\title{
Brownian and quantum motion
}

\section{A recent demonstration that a classical problem in brownian motion belies Dirac's relativistic equations of quantum mechanics is a reminder of many things that students should be taught.}

THE connection between brownian motion and quantum mechanics should be better known, at least to those brought up in the days before the full implications of Feynman's reformulation of quantum mechanics (in 1948) were fully appreciated, perhaps 15 years ago. That will be people's reaction to the neat argument, now published by B. Gaveau et al. (Phys. Rev. Lett. 53, 419; 1984) which sets out to provide a demonstration that Dirac's relativistic wave equation can be derived from a consideration of a simple classical problem in brownian motion. Others will however note that the authors include Mark Kac, now at the University of Southern California, Los Angeles, who first derived the Schrödinger equation from a brownian motion problem in 1959 , as well as $T$. Jacobson (University of Texas, Austin) and L.S. Schulman (Technion, Haifa), who have more recently made important contributions to similar problems.

The starting point for the argument is simple but evocative. Consider a particle moving in one dimension only at some fixed velocity, and let it suffer reversals of direction at random intervals, themselves distributed statistically according to some suitable law, most simply a Poisson distribution. Practitioners in brownian motion problems will readily write down expressions for the probability density (per unit length and per unit time) that the particle is at some place along the line at a certain time, and moving in one direction or the other. Most simply, there is a different function for each direction. The next step is to relate these probability densities to each other by taking account of the conservation in number of the particles moving along the line. The outcome is a set of differential equations which must be satisfied by the two probability densities. (In the sequel, these correspond to the two states of chirality or handedness which alone are accessible to relativistic Dirac particles in one dimension.) What the authors assert (and then prove) is that these equations are in an appropriate sense equivalent to Dirac's equation in one dimension. They then show that the same technique can be extended to the full relativistic problem (with three space dimensions).

The origin of this problem is intriguing, for it seems first to have appeared as an exercise for students in the elegent textbook Quantum Mechanics and Path
Integrals, by R. P. Feynman and A.R. Hibbs, published in 1965. There, users of the book are invited to calculate the evolution in time of a system consisting of a particle moving with the velocity of light in a single dimension, and suffering random reversals of direction. To Feynman and Hibbs, this would merely have been a test of what students had been told in the first thirty pages of the book. To Kac and company, the challenge has been to demonstrate that the connection between quantum mechanics and brownian motion that applies in non-relativistic mechanics also applies to Dirac's equation. That they succeed will not surprise those already in the know.

For the rest of us, there are several missing elements of which the least excusable is Feynman's use of the concept of the path integral. That, of course, is also familiar from classical mechanics. Since de Maupertuis and the principle of least action in the eighteenth century, it has been understood that the path that will actually be followed, and which is thus that dictated by the laws of motion, will be that for which the action (force multiplied by time) is least. For simple systems, forces can be calculated as a function of time from the function of the system called the lagrangian, essentially the difference between the kinetic and potential energies. Given initial and final states, and starting and finishing times, it is then possible to calculate (in principle at least) the action for all possible paths and to choose that for which the action is least.

The difference between this and quantum mechanics is that there is no unique path for the evolution of a system but that all possible paths must be counted. As Bohr and his disciples were always telling us, a photon confronted with two slits in a screen cannot be said to use one slit or the other, but both.

Each path is relevant; the only meaningful question to ask about the outcome is not where the particle finishes up, but the probability that it should finish up here or there and, to that end, that it is necessary to add together probability amplitudes (one for each possible path), taking account of plus and minus signs, and of imaginery as well as real parts, before calculating the probability (by squaring the amplitude). The essence of Feynman's quantum mechanics is a powerful prescription for calculating the evolution of the amplitude for each possible path. Inevitably, the outcome is a line integral. Although the integrals are not the same as those appearing in classical mechanics, their relationship is plain to see. In passing, it is worth asking whether quantum mechanics would ever be presented to students in other terms, necessarily less direct, if it were not that so much energy had been invested in teaching them to solve differential equations.

The connection with brownian motion is harder to discern. The formal resemblance with quantum mechanics is simple enough. Because the process is statistical, the most one can hope to do is to calculate the probability that a particle starting from one place on the line will finish up at another. Thus, if a particle is to start at one place and finish at another after a specified length of time, there is a large (even infinite) number of paths which it may traverse in doing so. The first to recognize this deeper relationship with Feynman's quantum mechanics - the existence of a multitude of paths - seems to have been $\mathrm{K}$. Ito, working on brownian motion in 1951 .

In reality a connection between the quantum mechanics of brownian motion gets even more specific. Put briefly, while the probability amplitudes in the brownian motion problem are all real and positive (and are thus simply additive), those which conspire in the calculation of Feynman's propagation functions are not. The quantum version of the calculations can be turned into the other version simply by replacing $i$ (the square root of minus 1 ) by minus 1 whenever it occurs. The similarity is even more striking when one sees it demonstrated that a specific path in Feynman's formulism can be boiled down to calculating the chances that a brownian particle makes its way along the same such trajectory by random process.

The implication is simply grasped. The propagation of the probability amplitude along an arbitrarily chosen trajectory is indeed to be likened to brownian motion. One of the quirks of that process, the fact that the average displacement of a particle is proportional to the square root of the time elapsed, accounts for some of the computational complexity of Feynman's formulism. Taken together, the whole box of tricks makes it intelligible that there should be a correspondence between the theory of quantum fields and that of phase transitions on a lattice. This is yet another part of the case for changing tack on teaching.
John Maddox 